\title{
Meshing Stiffness Calculation and Analysis of the Cycloidal Pump's Rotor
}

\author{
Liu M. T. and Xue Y. N. \\ Tianjin University of Science \& Technology, Tianjin, China
}

\begin{abstract}
In order to calculate the meshing stiffness of the cycloidal pump's rotor, a method of relationship that deduces two generating curtate epicycloid is proposed, the radius values of the inner rotor's actual tooth profile curvature was calculated and analyzed; Based on the formula of Hertz, the cycloidal pump rotor's meshing stiffness calculation formula is inferred, the changing curve of cycloidal pump rotor's meshing stiffness is made, some important parameters on its meshing stiffness are studied, the maximum value of meshing stiffness is when the outer rotor mesh with inner rotor's outer convex profile curve, and the minimum value is when the outer rotor mesh with the center of inner rotor's inner concave.
\end{abstract}

\section{Introduction}

Cycloidal pump is also called the cycloidal gear pump. Compared with other ordinary gear pumps, it has lots of advantages,such as compact structure, low noise, good self-priming performance, and suitable for high speed etc, and developed rapidly in recent years, widely used in food, textile, chemical, machinery and other industries. To accurately calculate the contact stress produced by the inner and outer rotor in cycloidal pump, the computation and analysis of the meshing stiffness is indispensable.

Reference [1] and [2] regard tooth inter-atomic forces and the displacement of mesh point in normal direction as direct ratio, which means the meshing stiffness is a fixed value. Due to the number of gear teeth of cycloidal pump rotor in the process of meshing is periodical change, its meshing force between the rotors is periodical. at the same time, the rotor tooth of the cycloidal pump's meshing stiffness is also cyclical change, solving the meshing stiffness of cycloidal pump rotors plays an important role in improving the transmission accuracy and analysis of the machine dynamic performance. Reference [3] formulates the mesh stiffness model of the RV reduction gears cycloidal-pin wheel's transmission pair based on Hertz formula and involute gear's transmission pair based on Shi Chuan formula, respectively. Reference [4] has an analysis and correction of single tooth meshing stiffness in reference [3], getting a new stiffness calculation formula; Reference [5] calculate ring-plate-type gear planetary drive's meshing stiffness by a tooth profile meshing analysis method taking clearance into account.

This paper researches the derivation relationship between two methods of generating short of epicycloid method, and then establishes the model of cycloidal pump rotor's meshing stiffness, getting each meshing tooth's meshing stiffness, and analyses its influencing factors.

\section{The formation of curtate epicycloid}

Cycloidal pump rotor pair is composed of a couple of internal meshing rotor as shown in Fig. 1. The outer rotor is internal gear and tooth profile curve is a certain number of equal distribution of circular arc curve; The inner rotor is external gear and its tooth profile curve is the equidistant curve of curtate epicycloid. There are two kinds of curtate epicycloid forming method: the forming method of two circle's inner gearing and the forming method of two circle's outer gearing [6], [7].

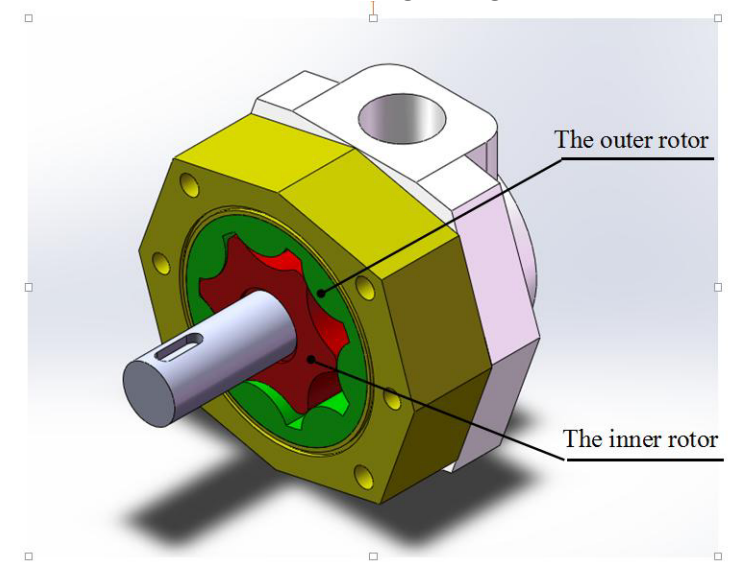

Figure 1. The cycloidal pump.

2.1 The relationship between two kinds of forming method of the cycloidal tooth profile. 
Two ways of forming cycloidal tooth profile are different, but as long as reaching a certain conditions, it can form the same curtate epicycloid. This paper proposes a new proof method on the basis of reference [6] and [7] as shown in Fig. 2:

Point $\mathrm{O}_{\mathrm{b}}, \mathrm{O}_{\mathrm{g}}$ is the center of circle 1 and circle 2 , respectively. $r_{b}, r_{g}$ is the radius of circle 1 and circle 2 , respectively, what' $\mathrm{s}$ more, $r_{b}>r_{g}$, circle 1 and circle 2 inscribes. Point $\mathrm{P}_{2}$ is the tangency point, draw a circle tangent to circle 1 (circle 2 ) go through point $P_{2}$; draw a circle using $\mathrm{O}_{\mathrm{b}}$ as the center and $R \mathrm{r}=R+r$ as the radius, the above two lines intersect at point $M$. It can regard the track of point $M$ as the track of point $M$ connected to the rolling outside in fixed plane trajectory, when a radius of $r_{b}$ rolling goes along the base circle with a radius of $r_{g}$ for pure roll.

Connect the line $O_{b} M$ and $P_{2} M$, respectively. Draw the parallel line of line $O_{b} M$ through point $\mathrm{O}_{\mathrm{g}}$; Draw the parallel line of line $O_{b} O_{g}$ through point $\mathrm{M}$, the above two lines intersect at point $\mathrm{O}$. It is easy to know that quadrilateral $\mathrm{OO}_{\mathrm{g}} \mathrm{O}_{\mathrm{b}} \mathrm{M}$ is a parallelogram. Draw a circle using $\mathrm{O}_{\mathrm{g}}$ as the center and $R$ as the radius; Draw a circle using $\mathrm{O}_{\mathrm{g}}$ as the center and $R$ as the radius; Therefore, it can also regard the track of point $M$ as the track of point $\mathrm{M}$ connected to the rolling outside in fixed plane trajectory, when a radius of $r$ rolling goes along the base circle with a radius of $R$ for pure roll, the eccentricity $e=r_{\mathrm{b}}-r_{\mathrm{g}}$.

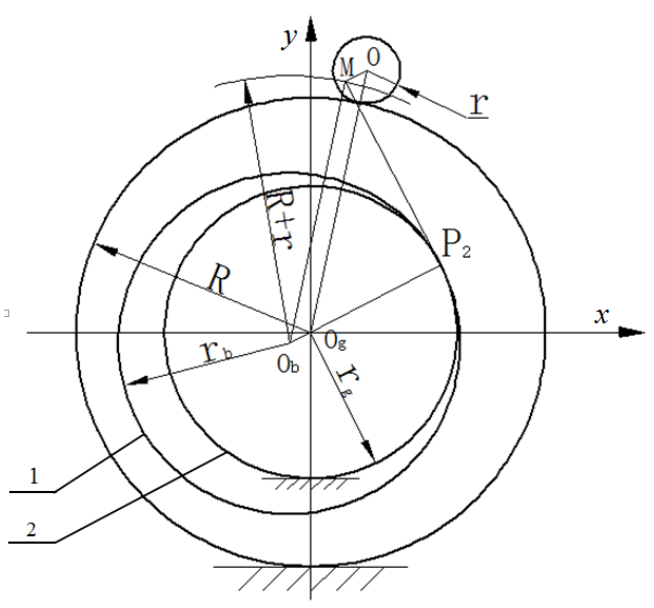

Figure 2. The Formation method of two kinds of curtate epicycloid.

Because of the quadrilateral $\mathrm{OO}_{\mathrm{g}} \mathrm{O}_{\mathrm{b}} \mathrm{M}$ is a parallelogram, two rolling radius $r_{b}, r$ and two base circle radius $r_{g}, R$ have the following relationships:

$$
\begin{gathered}
\triangle O_{g} P_{1} P_{2} \backsim \triangle O P_{1} M \backsim \triangle O_{b} M P_{2} \\
\frac{r_{b}}{\overline{O_{b} M}}=\frac{r_{b}}{R \mathrm{r}}=\frac{e}{r}=\frac{r_{g}}{R}
\end{gathered}
$$

Thus, the forming method of two circle's inner gearing and the forming method of two circle's outer gearing can be formed the same curtate epicycloid as long as meeting the above conditions.

\subsection{The curve equation of inner rotor}

The curve equation of the inner rotor's theoretic tooth profile can be expressed as:

$$
\left\{\begin{array}{l}
\mathrm{x}=R \mathrm{r} \sin \theta-\operatorname{esin}\left(\mathrm{Z}_{2} \theta\right) \\
\mathrm{y}=R \mathrm{r} \cos \theta-\operatorname{ecos}\left(\mathrm{Z}_{2} \theta\right)
\end{array}\right.
$$

The curve equation of the inner rotor's actual tooth profile can be expressed as:

$$
\left\{\begin{array}{l}
\mathrm{X}=R r \sin \theta-\mathrm{e} \cdot \sin \left(Z_{2} \theta\right)+a \cdot \frac{Z_{2} \mathrm{e} \sin \left(Z_{2} \theta\right)-R r \sin \theta}{\sqrt{Z_{2}^{2} \mathrm{e}^{2}+R r^{2}-2 R r \mathrm{Z}_{2} \cos \left(\mathrm{Z}_{1} \theta\right)}} \\
\mathrm{Y}=R r \cos \theta-\mathrm{e} \cdot \cos \left(Z_{2} \theta\right)+a \cdot \frac{Z_{2} \mathrm{e} \cos \left(Z_{2} \theta\right)-R r \cos \theta}{\sqrt{Z_{2}^{2} \mathrm{e}^{2}+R r^{2}-2 R r e \mathrm{Z}_{2} \cos \left(\mathrm{Z}_{1} \theta\right)}}
\end{array}\right.
$$

In the equation: $R \mathrm{r}$ is the outer rotor tooth profile uniform circular radius, $R \mathrm{r}=R+r, Z_{2}$ is the tooth number of outer rotor, $Z_{1}$ is the tooth number of inner rotor, $e$ is the eccentricity, $a$ is the outer rotor tooth profile radius, $\theta$ is the parameter.

Fig. 3 shows that the inner rotor's practical and theoretical contour line with $R \mathrm{r}=55 \mathrm{~mm}, Z_{2}=7, e=5 \mathrm{~mm}$, $a=20 \mathrm{~mm}$ :

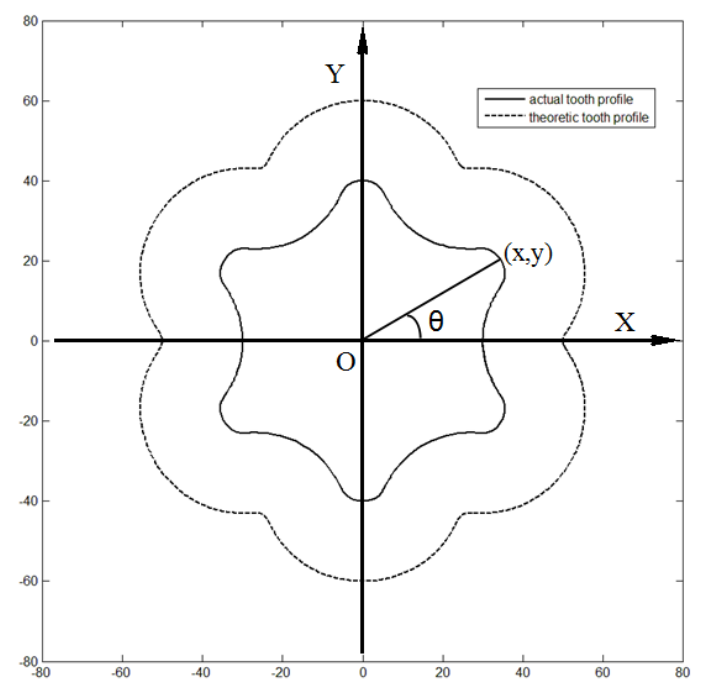

Figure 3. Practical and theoretical contour line of inner rotor

\subsection{The curvature radius of the inner rotor's actual contour}

The inner rotor's actual contour has outer convex and inner concave two cases as shown in Fig. 3. The curvature radius of the inner rotor's actual contour is equal to the curvature radius of the inner rotor's theoretic tooth profile plus the outer rotor tooth profile radius, then 
Here:

$$
\rho_{b}=\rho_{a}+a
$$

as cylinder. The tooth profile curve what the inner rotor is meshing has outer convex and inner concave two cases. The mesh transmission between the cycloidal rotors proceeds by line contact.In consideration of elastic deformation, the elastic deformation in the contact is a small area regional, the contact segment between inner rotor and outer rotor is arc. With regard to the mesh between inner rotor and outer rotor, it can assume that the deformation length is $2 L$, the tooth width is $B$ as shown in Fig. 5, so it can be calculated by Hertz formula ${ }^{[4]}$.

$$
\mathrm{L}=\sqrt{\frac{4 F}{\pi B} \cdot \frac{\frac{1-\mu_{1}^{2}}{E_{1}}+\frac{1-\mu_{2}^{2}}{E_{2}}}{\frac{1}{\rho}}}
$$

Here:

$$
\frac{1}{\rho}=\frac{1}{a} \pm \frac{1}{\left|\rho_{b}\right|}
$$

When the inner rotor meshing profile is outer convex, the inner rotor and outer rotor is external contact, where the \pm choose + .

When the inner rotor meshing profile is inner concave, the inner rotor and outer rotor is internal contact, where the \pm choose -

In the equation: $a$ is the radius of curvature of outer rotor at contact point, that's the outer rotor tooth profile radius, $F$ is the force at contact point, $\mu_{1}, \mu_{2}$ is the cycloid inner rotor and outer rotor material's poisson ratio, $E_{1}, E_{2}$ is the cycloid inner rotor and outer rotor material's elasticity modulus, $B$ is the rotor tooth width.

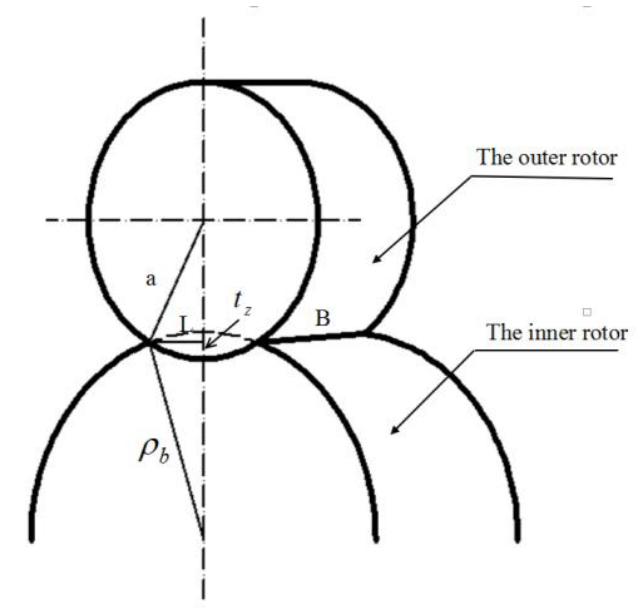

Figure 5. The extrusion deformation of the inner and outer rotor.

Due to the cycloid inner rotor and outer rotor material is the same, it can define $\mu=\mu_{1}=\mu_{2}, E=E_{1}=E_{2}$, then the (6), can be simplified as follows:

$$
\mathrm{L}=\sqrt{\frac{8 F \rho\left(1-\mu^{2}\right)}{\pi B E}}
$$

\subsection{The calculation of meshing stiffness}

The tooth profile curve what the outer rotor is meshing is the circular arc curve as shown in Fig. 1, and can be seen 


\subsubsection{The mesh between the outer rotor and inner rotor's outer convex profile curve}

The mesh between the outer rotor and inner rotor's outer convex profile curve. From the analysis of single outer rotor meshing stiffness in Fig. 5:

$$
\left(a-t_{Z}\right)^{2}+L^{2}=a^{2}
$$

In the equation: ${ }_{t_{Z}}$ is the extrusion deformation degree of the outer rotor in the radial direction.

$$
t_{Z}=a \cdot\left[1-\sqrt{1-\left(\frac{\mathrm{L}}{a}\right)^{2}}\right]
$$

Use Taylor formula at the point $\left(\frac{L}{a}\right)^{2}=0$, ignore the higher order infinitesimal, and put the (7), into it:

$$
t_{Z}=\frac{4 F \rho\left(1-\mu^{2}\right)}{\pi B E a}
$$

Therefore, single outer rotor meshing stiffness is obtained:

$$
C_{Z}=\frac{\pi B E}{4\left(1-\mu^{2}\right)} \cdot \frac{R r S^{3 / 2}+2 T a}{R r S^{3 / 2}+T a}
$$

Similarly, use Taylor formula at the point $\left(\frac{L}{\rho_{\mathrm{b}}}\right)^{2}=0$, ignore the higher order infinitesimal, and single inner rotor meshing stiffness is obtained:

$$
C_{\mathrm{b}}=\frac{\pi B E}{4\left(1-\mu^{2}\right)} \cdot \frac{R r S^{3 / 2}+2 T a}{T a}
$$

In conclusion, single inner rotor and outer rotor meshing stiffness can be written as

$$
K_{\text {outer }}=\frac{C_{b} C_{Z}}{C_{b}+C_{Z}}=\frac{\pi B E}{4\left(1-\mu^{2}\right)}
$$

\subsubsection{The mesh between the outer rotor and inner rotor's inner concave profile curve}

From the analysis of single outer rotor meshing stiffness in Fig. 5:

$$
\left(a-t_{Z}\right)^{2}+L^{2}=a^{2}
$$

In the equation: $t_{\mathrm{Z}}$ is the extrusion deformation degree of the outer rotor in the radial direction.

$$
t_{Z}=a \cdot\left[1-\sqrt{1-\left(\frac{\mathrm{L}}{a}\right)^{2}}\right]
$$

Use Taylor formula at the point $\left(\frac{L}{a}\right)^{2}=0$, ignore the higher order infinitesimal, and put the (7), into it:

$$
t_{Z}=\frac{4 F \rho\left(1-\mu^{2}\right)}{\pi B E a}
$$

Therefore, single outer rotor meshing stiffness is obtained:

$$
\mathrm{C}_{Z}=\frac{\pi B E}{4\left(1-\mu^{2}\right)} \cdot \frac{\operatorname{Rr} S^{3 / 2}}{\operatorname{Rr} S^{3 / 2}+T a}
$$

Similarly, use Taylor formula at the point $\left(\frac{L}{\rho_{\mathrm{b}}}\right)^{2}=0$, ignore the higher order infinitesimal, and single inner rotor meshing stiffness is obtained:

$$
\mathrm{C}_{\mathrm{b}}=\frac{\pi B E}{4\left(1-\mu^{2}\right)} \cdot \frac{\operatorname{Rr} S^{3 / 2}}{T a}
$$

In conclusion, single inner rotor and outer rotor meshing stiffness can be written as

$$
K_{\text {inner }}=\frac{C_{b} C_{Z}}{C_{b}+C_{Z}}=\frac{\pi B E}{4\left(1-\mu^{2}\right)} \cdot \frac{R \mathrm{r} S^{3 / 2}}{R \mathrm{r} S^{3 / 2}+2 T a}
$$

\subsubsection{The cycloid rotors meshing stiffness}

From the above, the cycloid rotors meshing stiffness $K_{S}$ is given as

$K=\left\{\begin{array}{l}\frac{\pi B E}{4\left(1-\mu^{2}\right)}, \text { mesh with the innerrotor's outer convex } \\ \frac{\pi B E}{4\left(1-\mu^{2}\right)} \cdot \frac{R \mathrm{r} S^{3 / 2}}{R \mathrm{r} S^{3 / 2}+2 T a}, \text { mesh with the innerrotor's inner concave }\end{array}\right.$

\section{Analysis Example}

According to (20), the rotor tooth width $B$, the cycloid rotor material's poisson ratio and elasticity modulus determines the maximum mesh stiffness between cycloid rotors. The rotor tooth width $B$ and meshing stiffness are proportional relationship, poisson ratio $\mu$ and elasticity modulus $E_{E}$ ratio is associated with the material of cycloid rotors. The majority of cycloid pumps' inner rotor teeth is 6 in practical application, this paper uses this kind of cycloidal pump to analyze. According to reference [8], the better value range of curtate ratio is from 0.50 to 0.75 . In this range, cycloidal pump has higher bearing capacity, less wear and tear. The selection of system parameters is shown in Table 1.

Table. 1 The design parameters

\begin{tabular}{ccc} 
Name & Symbol & Number \\
inner rotor's tooth number & $Z_{1}$ & 6 \\
outer rotor's tooth number & $Z_{2}$ & 7 \\
the eccentricity $(\mathrm{mm})$ & $e$ & 5 \\
the rotor tooth width $(\mathrm{mm})$ & $B$ & 44 \\
poisson ratio & $\mu$ & 0.25 \\
$\quad$ elasticity modulus $(\mathrm{Pa})$ & $E$ & $115 \mathrm{x} 109$ \\
the outer rotor tooth profile & $R r$ & $\mathrm{Z} 2 \mathrm{e} / \mathrm{k}$ \\
\hline uniform circular radius (mm)
\end{tabular}


As shown in Fig. 6, use the curtate ratio $\mathrm{k}=0.55$, $\mathrm{k}=0.64, \mathrm{k}=0.73$ as variables to make $a, b, c$ three curve. The theoretical value of the meshing stiffness cycle is $\pi / 3$, the meshing stiffness cycle in Fig. 6 is also $\pi / 3$. When the outer rotor mesh with inner rotor's outer convex profile curve, the meshing stiffness is constant, as well as the maximum value, the minimum value is when the outer rotor mesh with the center of inner rotor's inner concave, it's $\theta=k \frac{\pi}{3}+\frac{\pi}{6}, k=0,1, \ldots, 5$. In a cycle, the meshing stiffness remains the same in most of the time, its value varies considerably in other time, the greater the curtate ratio, the smaller the minimum value of meshing stiffness, and smaller variation as well.

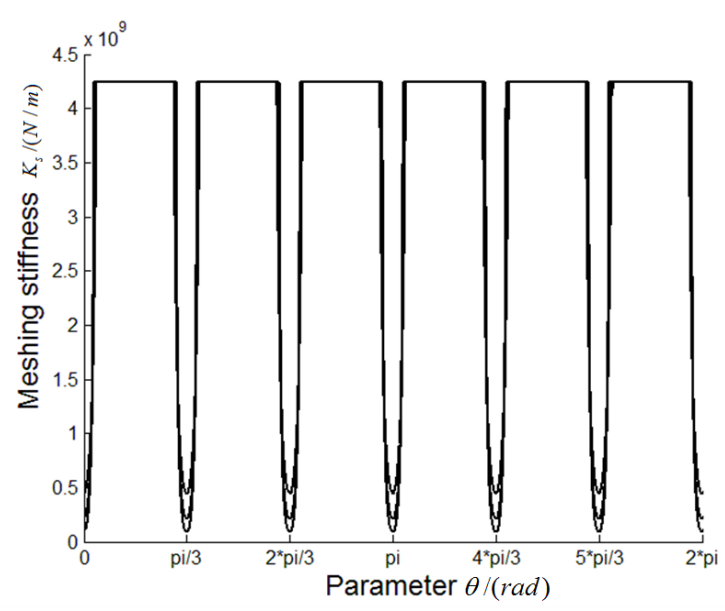

Figure 6. The meshing stiffness changing curve of different curtate ratio.

\section{Conclusions}

This paper researches the cycloidal pump, and gets the following conclusions:
Proposes a new method of relationship that deduces two generating curtate epicycloid.

Deduce the method of cycloid pump rotor's meshing stiffness, and get the rotor tooth width and meshing stiffness are proportional relationship.

The maximum value of meshing stiffness is when the outer rotor mesh with inner rotor's outer convex profile curve, and the minimum value is when the outer rotor mesh with the center of inner rotor's inner concave,it's $\theta=k \frac{\pi}{3}+\frac{\pi}{6}, k=0,1, \ldots, 5$. The greater the curtate ratio,the smaller the minimum value of meshing stiffness, and smaller variation as well.

\section{References}

1. Cao R., Wuhan University of Technology, (2006).

2. Wu D. , Zhang Y. C. , Coal Mine Machinery. 29, 6062, (2008).

3. Zhang D. W. ,Wang G. ,Huang T. , Journal of Mechanic Engineer. 37, 69-74, (2001).

4. Zhang Y. H., Xiao J. J. ,He W. D., Journal of Dalian Jiaotong University. 31, 20-23, (2010).

5. Shan L. J., Tian L. Y., Guan T. M. , He W. D. , Journal of Dalian Jiaotong University. 29,14-16, (2010).

6. Rao ZH. G., The Design of Planetary Transmission Mechanism, (National Defend Industry Press. 1994).

7. An Z. J., Yanshan University. (2000).

8. Mao H. Y. , Li G. X. , Liu H. T. , Hu Y. P. , Wang W. , Transactions of the Chinese Society of Agricultural Machinery. 37, 45-47, (2006). 